\title{
Episodic memory updating: The role of context familiarity
}

\author{
Almut Hupbach • Rebecca Gomez • Lynn Nadel
}

Published online: 7 June 2011

(C) Psychonomic Society, Inc. 2011

\begin{abstract}
We previously demonstrated that spatial context is a powerful reminder that can trigger memory updating (Hupbach, Hardt, Gomez, \& Nadel in Learning \& Memory, 15, 574-579 2008). In the present study, we asked whether the familiarity of the spatial context modulates the role of spatial context as a reminder. Since context familiarity can be easily manipulated in children, we chose 5-year-olds as study participants. In two experiments, we demonstrated robust memory-updating effects in children. Spatial context triggered incorporation of new information into old memories only when the context was unfamiliar. In highly familiar spatial environments (children's homes), spatial context did not initiate memory updating. Other reminders (the experimenter and a reminder question) became highly effective in familiar contexts. These findings shed further light on the specific conditions that trigger memory updating and support the view that the mechanisms underlying it are similar in children and adults.
\end{abstract}

Keywords Memory reconsolidation - Episodic memory Memory updating · Spatial context · Context familiarity · Memory development

Reactivation of "stable" long-term memories renders them malleable. In this state, memories can be modified, after which they need to be restabilized ("reconsolidated"). This phenomenon has been extensively studied for the

\footnotetext{
A. Hupbach $(\triangle)$

Department of Psychology, Lehigh University,

17 Memorial Drive East,

Bethlehem, PA 18015, USA

e-mail: hupbach@lehigh.edu

R. Gomez $\cdot$ L. Nadel

Cognition and Neural Systems, University of Arizona,

Tucson, AZ, USA
}

last decade in the animal domain, revealing the molecular and behavioral correlates of memory reconsolidation (for reviews, see Dudai, 2006; Nader, 2003; for molecular processes, see, e.g., Tronson \& Taylor, 2007). We previously developed a paradigm to study reactivation and updating effects in human episodic memory (Hupbach, Gomez, Hardt, \& Nadel, 2007) - that is, memories for events or episodes that have temporal and spatial signatures. In our paradigm, participants are asked to memorize a set of common objects. Later, participants are either reminded of this learning episode or not and then are asked to memorize an unrelated set of objects. When tested for memory of the first set, reminded participants intrude a significant number of Set 2 objects into their recall of Set 1. This is not the case for nonreminded participants, which underlines the importance of reactivation for memory modification. Moreover, the memory-updating effect is specific to Set 1. While reminded participants mistakenly assign Set 2 objects to Set 1 , they do not mistake the source of Set 1 memories (Hupbach, Gomez, \& Nadel, 2009). This specific asymmetric source error differs from the commonly reported bidirectional source confusion in the source memory literature. We have interpreted the asymmetric error as evidence for Set 1 memory modification. If participants in the reminder group find it difficult to assign objects to Set 1 or 2 because both sets were learned under similar conditions, one would expect them to equally confuse the source for Set 1 and Set 2 items. However, if Set 1 memory has been altered and now contains a subset of Set 2 items (or links to those items in memory), those Set 2 items will be ascribed to Set 1 (for further discussion, see Hupbach et al., 2009). However, no behavioral study can directly test whether the reminder indeed altered the original Set 1 memory (as assumed by the reconsolidation account), or whether the memories for Sets 1 and 2 coexist, and the specific encoding and retrieval conditions are what cause the 
false retrieval of a subset of Set 2 items, an explanation that is in line with the source-monitoring framework (Johnson, Hashtroudi, \& Lindsay, 1993) and current models of episodic memory retrieval (see below). According to the reconsolidation account, reactivation of Set 1 memory in Session 2 renders it labile and prone to modification. When Set 2 objects are presented while Set 1 memory is in this labile state, Set 2 objects become integrated into Set 1 memory through a timedependent restabilization (reconsolidation) process, resulting in an altered (or updated) memory for Set 1.

Since the reconsolidation account is largely a neurobiological model explaining animal data, the cognitive processes involved in human memory updating remain unspecified. In contrast, computational models can be used to develop detailed descriptions of the mechanisms involved in memory encoding and retrieval. Sederberg, Gershman, Polyn, and Norman (2011) have recently successfully simulated our memory-updating findings (Hupbach et al., 2007; Hupbach et al., 2009) using the temporal context model (TCM; Howard \& Kahana, 2002; Sederberg, Howard, \& Kahana, 2008), providing a plausible specification of the processes underlying our findings at the computational level. In TCM, encoding binds an item to its current temporal context. Temporal context is represented as a recency-weighted average of past experience. Later recall of an item cues the recall of the contextual state in which it was presented (via item-context associations). This in turn triggers the recall of items that share aspects of the temporal context with the currently active item (via context-item associations). With regard to our memory-updating effect, Sederberg et al. (2011) assume that the reminder in Session 2 triggers reinstatement of the Set 1 temporal context. This will cause Set 2 items to be associated with the Set 1 context as well as with their unique Set 2 context. When participants are asked to recall Set 1 , the Set 1 temporal context will be reinstated, and since this context is associated not only with Set 1, but also with Set 2, some Set 2 items will be recalled. In the no-reminder condition, the Set 1 context is not associated with Set 2 items, and hence, Set 2 items do not intrude into Set 1 recall. Additionally, because of the asymmetrical nature of forward cuing in time, the model can also explain the asymmetry of intrusions (Set 1 recall leads to intrusions of Set 2 items, and not vice versa): While Set 2 items are associated with a temporal context that contains features of the Set 1 context, the context layer of Set 1 items does not contain context features of Set 2, because Set 1 was presented before Set 2. Although TCM provides a satisfying mechanistic account of the basic memoryupdating findings, several issues remain unresolved. For instance, the model's emphasis on temporal context stands in contrast to the importance of spatial context for memory updating (Hupbach, Hardt, Gomez, \& Nadel, 2008). As noted by the Sederberg et al. (2011), the model cannot explain why spatial context plays a special role in triggering updating (see below).

In a study specifically investigating the nature of the reminder that best reactivates memory, we found that spatial context is the primary factor initiating memory updating: Only if participants learned the second set in the same room as the first set did they show incorporation of Set 2 items into Set 1 memory. Encountering the same experimenter in the second session and/or answering a reminder question before learning the second set had no such effect when these took place in a different room, nor were these reminders additive with respect to spatial context (Hupbach et al., 2008).

In our previous studies, participants (undergraduate college students) were tested in our laboratory in a room that they had never visited prior to participation in the experiment; hence, the spatial context was unfamiliar. Would a familiar context also initiate memory updating? Contexts help organisms predict what will happen in specific places. Since a familiar context is associated with many different experiences or events, this might diminish its predictive value, and hence, specific episodes might not be reactivated when the context was revisited.

Although there is a large body of literature on the spatial context dependency of memories (for a meta-analysis, see Smith \& Vela, 2001), no study involving human participants has looked at how context familiarity affects memory. Animal studies have suggested that novel and familiar contexts are processed differently; for instance, rats' principal cells in the hippocampus show differential firing patterns in response to novel and familiar environments (e.g., Lever et al., 2010). Behavioral studies on contextual conditioning in rats show that extensive familiarization with a context can retard aversion conditioning to that context (latent inhibition; Hall \& Symonds, 2006; Hall, Symonds, \& Rodriguez, 2009). During the nonreinforced preexposure, rats learn that the spatial context does not predict a specific event. Two cognitive phenomena, although not spatial in nature, also illustrate that cues become less effective when they are associated with many outcomes. The cueoverload principle (Watkins \& Watkins, 1975) states that the probability of recalling a target declines with the number of targets sharing the same retrieval cue. The fan effect (Anderson, 1974) refers to the finding that as more facts are studied about a concept, it takes longer to retrieve a particular fact. Given participants' many preexperimental associations between that context and various events and daily routines, one could hypothesize that a familiar context might not be effective in reactivating a specific memory.

We chose to study the effects of context familiarity in a sample of children because children usually live in familiar home environments, whereas college students 
vary with regard to the familiarity of their home setting (many of them have just moved from their childhood home), and most of them are not familiar with our laboratory test rooms. Additionally, we were interested in seeing whether our memory-updating effect would generalize to a population that demonstrates difficulty with source attribution.

To our knowledge, there are no developmental studies that specifically address memory reconsolidation, but our paradigm is similar to the misinformation paradigm (see, e.g., Loftus, 2005, for a review) and to retroactive interference studies (e.g., Postman \& Underwood, 1973): Participants first encode an event or set of items and are subsequently presented with additional information that, in the case of interference and the misinformation effect, differs from the previously learned information. When later asked to recall the original material, participants show impaired recall and/or replace some of the original information with the incorrect postevent information. Indeed, it has recently been suggested that the memory reconsolidation process being studied in rat models could underlie these human memory effects (Hardt, Einarsson, \& Nader, 2010). One critical difference in our paradigm is that we present two sets of unrelated objects that do not directly compete. This could explain why our participants incorporate new items into old memories, instead of replacing old with new information, as is the case for the misinformation and retroactive interference effects.

Few studies have directly tested retroactive interference in children. While an early study with 4-, 5-, and 8-yearolds showed an age-related increase in retroactive interference (Koppenaal, Krull, \& Katz, 1964), Howe (1995) found large and age-invariant interference effects in preschool and kindergarten children and identified the locus of the interference effects at storage. Lee and Bussey (2001) reported similar age-invariant retroactive interference effects for 4- and 7-year-olds, and, like Howe (1995), found that retroactive interference effects were independent of initial levels of learning.

In contrast to the age-invariant retroactive interference effects, younger children are generally more susceptible to misinformation than are older children and adults (e.g., Bruck \& Ceci, 1999; but see Brainerd, Reyna, \& Ceci, 2008, for developmental reversals). The greater susceptibility of younger children has been related to their poor source memory. Significant improvements in source monitoring take place between the ages of 3 and 8 (see the review by Roberts, 2002). For instance, Drummey and Newcombe (2002) taught 4-, 6-, and 8year-olds facts presented by two different sources, the experimenter or a puppet. In a subsequent test, 4-yearolds could not remember whether they had learned the facts inside or outside the experimental context. The 6and 8-year-olds knew which facts they had acquired during the experiment but had difficulty remembering which ones had been introduced by the experimenter and which by the puppet. In another study, 7- to 8-year-old children showed adultlike memory performance in a picture recognition test but impaired performance when asked to remember whether the picture had been presented in green or red during encoding (Cycowicz, Friedman, Snodgrass, \& Duff, 2001). Further, it has been shown that age-appropriate cues facilitate source monitoring and reduce suggestibility in 3- to 7-year-olds (Bright-Paul, Jarrold, \& Wright, 2005).

Taken together, studies on retroactive interference and misinformation suggest that memory-updating mechanisms are functional in children, and that memory might be even more malleable in children than in adults. Based on the assumption that similar mechanisms underlie retroactive interference, the misinformation effect, and our updating effect, we hypothesized that the 5-year-olds in our study would show reminder-induced modification of the original memory, much like adults. However, since children have greater difficulty remembering the source of information, they might have general difficulties remembering which objects were presented on which day, and hence might not only show intrusions from Set 2 into Set 1, but also from Set 1 into Set 2. This was tested in Experiment 2B.

\section{Experiment 1}

The first experiment is a replication of our previous finding of context-dependent updating of episodic memories (Hupbach et al., 2008) with a sample of 5-year-old children. The adults in our previous study were tested in our laboratory, which they had never visited prior to participation in the experiment; hence, the specific spatial context was unfamiliar, while the more general spatial context (the Psychology Building) was familiar. To implement the same conditions with 5-year-olds, we tested them in their daycare and preschool setting (a highly familiar general context) in a place that they did not visit on a regular basis. If a rather unfamiliar spatial context reactivates a specific memory in children as in our adult study, we should see intrusions from Set 2 into Set 1 in the group that learns Set 2 in the same spatial context as Set 1 (context reminder), but not in the group that switches contexts between Sessions 1 and 2 (no reminder).

\section{Method}

Design and participants A total of 24 children were randomly assigned to the context-reminder or the noreminder group, but 1 child in the context-reminder and 3 children in the no-reminder group missed one or more 
sessions and were therefore excluded from the final data set. The final sample consisted of 20 5-year-olds (10 girls, 10 boys, mean age $=5$ years, 1 month; range $=$ 4 years, 6 months, to 5 years, 6 months), of whom 11 were in the context-reminder group (6 girls) and 9 in the no-reminder group (4 girls).

Materials The to-be-remembered (Set 1) and interference (Set 2) materials were each eight unrelated objects: Set 1-toy car, cup, flashlight, glue, key, sock, sunglasses, tennis ball; Set 2-balloon, box, candle, crayon, feather, sponge, spoon, toothbrush. In Session 1, the objects were sorted into a blue basket.

Procedure The three sessions took place on three consecutive days at a daycare facility. Children participated one at a time outside of their classroom in an unfamiliar space at the daycare center, one that was not visited during the children's daily routines.

During Session 1, the experimenter pulled out one object at a time from a bag and placed it into a distinctive blue basket. The child was asked to name each object and to pay close attention so that he or she could remember the objects later. After all eight objects had been placed into the basket, the experimenter hid the basket and asked the child to remember as many objects as possible. This procedure was repeated until the child recalled at least six of the eight objects or until a maximum of four learning trials was reached.

During Session 2, half of the children were seen in the same spatial context (context reminder) and half were seen in a different unfamiliar location at the daycare center (no reminder). All children worked with a different experimenter. The experimenter did not mention the previous day, the basket, or the objects. All children were asked to learn the second set of objects. The procedure differed from that of Session 1 so that the task itself would not serve as a reminder. All objects were placed in front of the child. The child was asked to name each of the objects and was given $30 \mathrm{~s}$ to study them. The experimenter then removed the objects and asked the child to recall as many as possible. The procedure was repeated until the child recalled six of the eight objects, for a maximum of four learning trials.

During Session 3, the experimenter from Session 1 administered the memory test in the spatial context used during Session 1. The empty blue basket was placed in front of the child, and the child was asked to remember as many objects as possible from Session 1. After a 30-s delay, the experimenter asked the child to recall the objects again. This was repeated for four consecutive recall trials to encourage children to remember as many items as possible.
Results

Initial learning In order to compare initial levels of learning of the Set 1 and Set 2 objects in the contextreminder and no-reminder groups, we analyzed the final numbers of items recalled in Sessions 1 and 2 with a $2 \times$ 2 mixed ANOVA with Session (1 vs. 2) as the withinsubjects factor and Group (context vs. no reminder) as the between-subjects factor. No significant effects were detected ( $F \mathrm{~s} \leq 1.72, p \mathrm{~s} \geq .21)$; that is, initial levels of learning were comparable in both groups and across Sets 1 and 2.

Recall The mean percentages of objects recalled from Set 1 and the mean percentages of objects falsely recalled from Set 2 (both averaged over the four retrieval trials) are displayed in Fig. 1.

Set 1 recall The numbers of objects recalled from Set 1 were analyzed with a $2 \times 4$ mixed ANOVA with Group (context vs. no reminder) as the between-subjects factor and Trial (1-4) as a within-subjects factor. No significant effects were detected $(F \mathrm{~s} \leq 2.17, p \mathrm{~s} \geq .13)$. Thus, in contrast to what has been reported by Howe and Brainerd (1989), children's recall performance did not improve across trials.

Intrusions A 2 (group) $\times 4$ (trial) ANOVA with the numbers of objects falsely recalled from Set 2 as the dependent variable revealed a significant effect of group $[F(1,18)=11.60, M S E=3.67, p<.01 ;$ all other $F \mathbf{S}<1]:$ Children in the context-reminder group intermixed significantly more objects from Set 2 into Set 1 recall $(M=22.2 \%, S D=13.1 \%)$ than did children in the noreminder group $(M=3.8 \%, S D=10.3 \%)$. Because Set 1 recall was negatively correlated with Set 2 intrusions

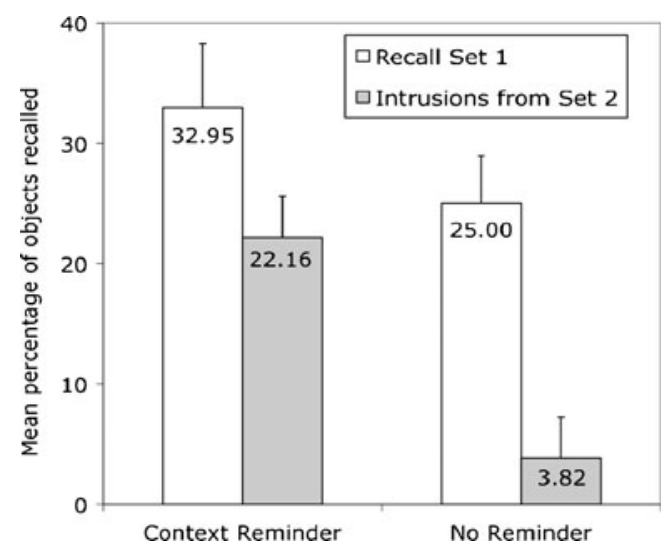

Fig. 1 Mean percentages of objects correctly and falsely recalled in the context-reminder and no-reminder groups in Experiment 1 (unfamiliar spatial context). Error bars represent standard errors of the means. Note: Children were asked to recall objects from Set 1. Objects that were falsely recalled from Set 2 are labeled as Intrusions 
(see below), we additionally ran an ANCOVA with the number of objects falsely recalled from Set 2 (averaged over the four recall trials) as the dependent variable and Set 1 recall (averaged over the four recall trials) as the covariate. The covariate was significant $[F(1,17)=4.62$, $M S E=.77, p=.05]$, but most importantly, the group effect remained significant $[F(1,17)=17.43, M S E=.77, p<.01]$.

Because the no-reminder group showed few intrusions (floor effects) and the variance was higher in the reminder than in the no-reminder group, we ran an additional nonparametric test with the mean number of intrusions recalled by each participant (averaged over the four test trials) serving as the dependent variable. A Mann-Whitney $U$ test detected significant group differences $(U=12.00, p<.01)$.

Additional analyses for the context-reminder group In order to better understand when intrusions occur and how they relate to recall of Set 1, we ran some additional analyses for the context-reminder group only: (1) There was a significant negative correlation between the mean number of objects correctly recalled from Set 1 and the mean number of objects falsely recalled from Set 2 (both averaged over the four recall trials; $r=-.79, p<.01$ ), showing that greater target recall was associated with fewer intrusions. (2) In order to analyze how intrusion levels developed across trials, we calculated the proportion of intrusions among the total number of recalled objects (including Set 1 recalls and Set 2 intrusions) for each recall trial. The proportions of intrusions did not change across trials $\left(M_{\text {Trial1 }}=.38, M_{\text {Trial2 }}=.43, M_{\text {Trial3 }}=.41, M_{\text {Trial4 }}=.44\right.$; $F<1$ ). (3) The output order for Set 1 and Set 2 objects was analyzed for the first retrieval trial only, because subsequent retrieval attempts were most likely influenced by previous trials, and therefore do not provide a pure measure of output position. We calculated the relative position of a recalled item by calculating output percentile scores following the procedure introduced by Bjork and Whitten (1974): If a child recalled a total number of $N$ objects, the $i$ th object recalled was given a percentile score of $(i / N) \times 100$. For instance, the first of four items recalled results in a score of 25 , and the fourth of five items recalled results in a score of 80. This procedure has the advantage of normalizing the object's output positions with regard to the number of objects recalled. Furthermore, differences in mean output percentiles for the two sets would indicate whether one set was recalled before the other, with lower percentiles reflecting earlier recall. Mean output percentiles did not differ for items from Sets 1 and $2\left(M_{\text {Set } 1}=64 \%, M_{\text {Set } 2}=69 \% ; t<1\right)$. (4) As an additional measure of output order, we computed whether the first item recalled was a Set 1 or Set 2 object (probability of first recall; Howard \& Kahana, 1999). This measure speaks to the strongest item associated with Set 1. Eight children recalled a Set 1 object first, and 3 children recalled a
Set 2 object first. This difference was not statistically significant $\left(\chi^{2}=2.27, p=.13\right)$

\section{Discussion}

Experiment 1 replicates our previous finding with adults (Hupbach et al., 2008). When the second learning experience took place in the same spatial context as a related first learning experience, the second memory intruded into the first. Very few intrusions were observed when the two learning episodes took place in different spatial settings. This shows that 5-year-old children update their memory when a later learning episode takes place in the same environment as a previous learning episode. We have interpreted the finding of context-dependent intrusions as demonstrating a special role for spatial context in the updating of episodic memories: In our view, it provides a scaffold binding the elements of experiences occurring within the context. Later episodes that occur in the same spatial context will reactivate the old episode and update its content by binding new elements (Hupbach et al., 2008).

Experiment 1 showed a negative relationship between target recall and intrusion levels, suggesting that stronger memories are less likely to be updated. This is in line with both animal work showing that memory strength modulates fear reconsolidation (Wang, de Oliveira Alvares, \& Nader, 2009) and preliminary data from our lab showing that increasing the strength of Set 1 memory by overtraining participants on Set 1 results in a reduction of intrusions (Hupbach, Weiss, Gomez, \& Nadel, 2011).

As was pointed out in the introduction, although TCM provides a reasonable mechanistic account of the memoryupdating phenomenon, it cannot explain certain behavioral findings. TCM predicts that when participants are cued to recall Set 1 in Session 3, Set 1 objects should be recalled before Set 2 objects, because the Set 1 temporal context (reinstantiated when children are instructed to recall Set 1) is more strongly associated with Set 1 than with Set 2 objects (Sederberg et al., 2011). This expected output pattern was not confirmed by the analysis of the mean output percentiles (Bjork \& Whitten, 1974): Set 1 and Set 2 output positions did not differ, a finding that cannot be readily explained by TCM. However, if Set 2 items are integrated into Set 1 memory, as assumed by the reconsolidation account, their recall position would not be expected to follow a specific output pattern. Although the result was not statistically significant, the analysis of the first item recalled during Set 1 recall (Howard \& Kahana, 1999) did show a numerical advantage for Set 1 over Set 2 items. Since this measure only considers one item per participant, we consider it a weaker test of output 
order than the Bjork and Whitten measure. More data will be needed to adequately test the predictions of TCM in this condition.

Experiment 2 tested whether a highly familiar spatial context initiates memory reactivation and updating.

\section{Experiment 2A}

In Experiment 2, we tested children in their homes. If a familiar spatial context fails to trigger updating of episodic memories, children who learn about both sets of objects in the same spatial context should not show intrusions from the second set into the first set. This was tested in Experiment 2A. However, other cues might contribute to updating in a familiar context. In our previous study demonstrating context-dependent updating in adults in an unfamiliar context, we tested the contributions of two other reminders, the experimenter and a reminder question (Hupbach et al., 2008). These reminders, in isolation and in combination, failed to trigger memory updating in an unfamiliar context: Set 2 did not intrude into Set 1 when the experimenter was the same during Sessions 1 and 2, or when a reminder question was asked before learning Set 2 . However, when the spatial context is highly familiar, these two reminders might contribute to reactivation and updating. Thus, children learned the second set in the same spatial context as the first, and they either were tested by the same experimenter on all 3 days and were asked a reminder question before learning Set 2 in Session 2 (three-component reminder) or were tested by a different experimenter in Session 2 and received no reminder question (spatial-context reminder).

\section{Method}

Design and participants Twenty-four 5-year-olds (16 girls, 8 boys, mean age $=4$ years, 10 months; range $=4$ years, 0 months, to 5 years, 8 months) participated in the experiment. Twelve children each were randomly assigned to the three-component reminder (8 girls) or the contextreminder group ( 8 girls). Table 1 gives an overview of the experimental conditions utilized in Experiment 2.

Materials and procedure The exact same materials and procedure were used as in Experiment 1, with the exception that the three experimental sessions took place at the same spatial location within the children's homes. In the threecomponent reminder group, the same experimenter visited the children and asked the following reminder question before Set 2 learning: "Can you describe to me what we did with the blue basket yesterday?" We intended for children to describe the general procedure and not to recall individual items, in order to avoid testing effects. None of the children recalled any items spontaneously.

\section{Results}

Initial learning The final numbers of items recalled in Sessions 1 and 2 were analyzed with a $2 \times 2$ mixed ANOVA with Session (1 vs. 2 ) as the within-subjects factor and Group (three-component vs. context reminder) as the between-subjects factor. No significant effects were detected $(F \mathrm{~s} \leq 2.85, p \mathrm{~s} \geq .11)$, showing that initial levels of learning were comparable in both groups and across Sets 1 and 2 .

Recall The mean numbers of objects recalled from Set 1 and the mean numbers of objects falsely recalled from Set 2 are displayed in Fig. 2.

Set 1 recall A 2 (group) $\times 4$ (trial) ANOVA of the numbers of objects recalled with Group (three-component vs. context reminder) as the between-subjects factor and Trial $(1-4)$ as a within-subjects factor revealed a significant effect of trial $[F(3,66)=3.48, M S E=.55, p=.02$; linear contrast: $F(1,22)=4.88, M S E=.90, p=.04]$ and a trial by group interaction $[F(3,66)=3.25, M S E=.55, p=.03]$. The analysis of the simple effects showed that recall performance did not change in the three-component reminder group $(F<1)$ but did increase across trials in the contextreminder group $[F(3,66)=6.19, M S E=.55, p<.01]$. This improvement had not been found in Experiment 1, but it replicates previous studies (cf. Howe \& Brainerd, 1989).

Intrusions A 2 (group) $\times 4$ (trial) ANOVA with the numbers of objects falsely recalled from Set 2 as the dependent variable revealed a significant effect of group $[F(1,22)=$ 23.88, $M S E=3.30, p<.01$; all other $F \mathrm{~s} \leq 1.32, p \mathrm{~s} \geq .27]$ : Children in the three-component reminder group intruded significantly more objects from Set 2 into Set 1 recall $(M=28.4 \%, S D=13.6 \%)$ than did children in the context-reminder group $(M=5.2 \%, S D=8.4 \%)$. A MannWhitney $U$ test of the mean number of intrusions averaged over the four recall trials confirmed this result $(U=9.50$, $p<.01)$.

Additional analyses for the three-component reminder group (1) In contrast to Experiment 1, mean recall levels were not correlated with mean intrusion levels $(r=-.09$, $p=.78$ ). (2) As in Experiment 1, the proportions of intrusions among the total number of recalled objects (including Set 1 recalls and Set 2 intrusions) did not change across trials $\left(M_{\text {Trial1 }}=.43, M_{\text {Trial2 }}=.48, M_{\text {Trial3 }}=.48, M_{\text {Trial } 4}=.53 ; F<1\right)$. (3) Mean output percentiles were lower for Set $1(M=46 \%)$ 
Table 1 Overview of the experimental conditions used in Experiment 2 (familiar context)

\begin{tabular}{|c|c|c|c|}
\hline Experimental Group & Session 1 & Session 2 & Session 3 \\
\hline \multicolumn{4}{|l|}{ Experiment $2 \mathrm{~A}$} \\
\hline Three-component reminder & Encoding Set 1 & Same experimenter, Reminder question, Encoding Set 2 & Recall Set 1 \\
\hline Context reminder & Encoding Set 1 & Different experimenter, No reminder question, Encoding Set 2 & Recall Set 1 \\
\hline \multicolumn{4}{|l|}{ Experiment $2 \mathrm{~B}$} \\
\hline Set 2 recall & Encoding Set 1 & Same experimenter, Reminder question, Encoding Set 2 & Recall Set 2 \\
\hline Immediate recall & Encoding Set 1 & Same experimenter, Reminder question, Encoding Set 2, Recall Set 1 & - \\
\hline
\end{tabular}

than for Set 2 items $(M=82 \%)$, showing that Set 1 items were recalled earlier than Set 2 items $[t(11)=6.13, p<.01]$. (4) Additionally, all children recalled a Set 1 object as their first item during recall.

\section{Discussion}

Experiment 2A shows that a familiar spatial context on its own does not function as a reminder: When children were tested at their homes and learned the two sets of objects in the same spatial location, very few Set 2 objects were falsely recalled in Session 3, in which children were asked to recall Set 1 objects. However, in the group that had two additional reminder components (the same experimenter administered Session 2 and asked a reminder question), children showed a high number of intrusions from Set 2 into Set 1. Thus, in a highly familiar spatial environment, other reminders become effective. Why these reminders might be effective in familiar but not in unfamiliar contexts will be discussed in the General Discussion.

In contrast to Experiment 1, the analysis of the output order showed that children in the three-component reminder group recalled Set 1 objects earlier in the recall process than

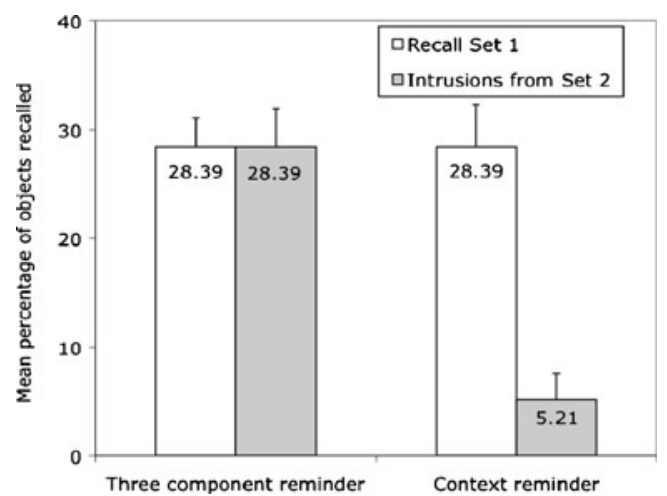

Fig. 2 Mean percentages of objects correctly and falsely recalled in the three-component reminder and context-reminder groups in Experiment 2A (familiar spatial context). Error bars represent standard errors of the means. Note: Children were asked to recall objects from Set 1 . Objects that were falsely recalled from Set 2 are labeled as Intrusions
Set 2 objects, and that all children recalled a Set 1 object as their first item during recall. Thus, TCM's predictions of earlier recall of Set 1 than Set 2 items were confirmed in familiar environments (Exp. 2A), in which reactivation and updating are initiated by the experimenter and reminder question, but not in unfamiliar ones, in which the spatial context is the trigger for updating (Exp. 1). Future research needs to determine whether the familiarity of the environment and the different reminders that are effective in each environment lead to fundamental differences in memory updating.

\section{Experiment 2B}

To further evaluate the nature of the updating effect in the three-component reminder group, we ran an additional experiment that included two control groups.

In order to test whether updating is time dependent, we asked children to recall the objects from the first set immediately after they had learned the second set in Session 2 (immediate recall group). Considerable work with animal models has shown that memory reconsolidation is a time-dependent process, because it requires the synthesis of new proteins (e.g., Nader, 2003). In animal studies, memory reconsolidation is only observed after a delay of about $4 \mathrm{~h}$. We observed the same effect in adults in our paradigm: Immediate retesting after acquisition of Set 2 fails to yield intrusions from Set 2 into Set 1 (Hupbach et al., 2007). Thus, if the effect we observed in children reflects a reconsolidation process, we should not see intrusions from Set 2 into Set 1 when children are tested immediately after learning Set 2.

Additionally, as noted above, young children often have source memory problems (Cycowicz et al., 2001; Drummey \& Newcombe, 2002). In order to determine whether intrusions from Set 2 into Set 1 reflect a general source discrimination problem (i.e., children have difficulties remembering which objects were presented on which day), we asked another group of children to recall the second set instead of the first set in Session 3. In our previous studies with adults, we found no evidence for general 
source discrimination problems (Hupbach et al., 2007; Hupbach et al., 2009). However, if children have a source memory problem overall, they might not only intrude Set 2 objects into Set 1 recall, as was shown in Experiment $2 \mathrm{~A}$, but also intrude Set 1 objects into Set 2 recall.

\section{Method}

Participants Twenty 5-year-olds participated in the experiment (10 girls, 10 boys, mean age $=5$ years, 1 month; range $=4$ years, 2 months, to 5 years, 10 months). Of these children, 8 were randomly assigned to the immediate recall group (4 girls); they were reminded of Session 1, through the use of the same experimenter and a reminder question, but were asked to recall Set 1 immediately after learning Set 2 in Session 2. Recruitment of participants in this group was stopped after analyzing the data of 8 children, because all children uniformly showed very low intrusions. Another 12 children were randomly assigned to the Set 2 recall group (6 girls). Those children received a reminder (same experimenter and reminder question) in Session 2 but were asked to recall Set 2 instead of Set 1 in Session 3.

Material and procedure The exact same materials were used as in Experiments 1 and 2A. The procedure was identical to that of Experiment 2A, with the exception that (a) in the immediate recall group, Session 3 followed Session 2 immediately (i.e., there was no time delay between Sessions 2 and 3), and (b) in the Set 2 recall group, children were asked to recall Set 2 instead of Set 1 in Session 3 (administered $24 \mathrm{~h}$ after Session 2, as in Exp. 2A).

\section{Results}

Because both groups in Experiment 2B served as controls for the three-component reminder group of Experiment 2A, both groups were separately compared to this group with regard to the number of objects recalled and intruded.

Comparison between the immediate recall group and the three-component reminder group (Exp. 2A)

Initial learning The final numbers of items recalled in Sessions 1 and 2 were comparable in both groups and sets of objects $\left(F_{\mathrm{s}} \leq 1.51, p \mathrm{~s} \geq .24\right)$.

Set 1 recall The immediate recall group recalled on average $35.2 \%(S D=15.5 \%)$ of the Set 1 objects (three-component reminder group, Exp. $2 \mathrm{~A}: M=28.4 \%, S D=9.3 \%$ ). A 2 (group) $\times 4$ (trial) ANOVA with the number of items recalled from Set 1 as the dependent variable showed no significant effects $(F \mathrm{~s} \leq 1.21, p \mathrm{~s} \geq .29)$.
Intrusions A 2 (group) $\times 4$ (trial) mixed ANOVA of the numbers of Set 2 intrusions revealed a significant effect of group $[F(1,18)=32.35, M S E=2.90, p<.01]$ : Children in the immediate recall group showed fewer intrusions $(M=0.8 \%, S D=1.4 \%)$ than did children in the three-component reminder group in Experiment 2A $(M=28.4 \%, S D=13.6 \%)$. A Mann-Whitney $U$ test of the mean numbers of intrusions averaged over the four recall trials confirmed this group difference $(U=0, p<.01)$.

Comparison between the Set 2 recall group and the three-component reminder group (Exp. 2A)

Initial learning The final numbers of items recalled in Sessions 1 and 2 were comparable in both groups and sets of objects $(F<1)$.

Recall A 2 (group) $\times 4$ (trial) ANOVA of the numbers of objects recalled in Session 3 revealed a significant effect of group $[F(1,22)=12.89, M S E=3.01, p<.01]$ : The Set 2 recall group recalled significantly more objects $(M=$ $44.3 \%, S D=12.2 \%)$ than the three-component reminder group (Exp. 2A), who had recalled Set 1 in Session 3 ( $M=$ $28.4 \%, S D=9.3 \%)$.

Intrusions A 2 (group) $\times 4$ (trial) ANOVA of the numbers of intrusions showed that the Set 2 recall group had significantly fewer intrusions (Set 1 into Set 2 recall, $M=8.6 \%, S D=11.8 \%)$ than did the three-component reminder group [Set 2 into Set 1 recall, $M=28.4 \%, S D=$ $13.6 \% ; F(1,22)=14.58, M S E=4.13, p<.01]$. A MannWhitney $U$ test of the mean numbers of intrusions averaged over the four recall trials also detected significant group differences $(U=19.00, p<.01)$.

\section{Discussion}

Memory performance in the control groups largely replicated what we have previously found with adults (Hupbach et al., 2007). Intrusions from Set 2 into Set 1 emerged over time and were not observed in a test immediately following Set 2 presentation. This pattern of results is in line with the reconsolidation interpretation of the intrusion effect. Alternatively, children might use a "rejection diagnostic" in order to avoid intrusions; that is, they might have rejected Set 2 objects that come to mind on the basis of recollecting that they had been encoded in the same session earlier (Sederberg et al., 2011). That it would be easier for children to adopt this strategy in the immediate recall (Exp. 2B) than in the delayed recall (Exp. 1) can be explained by the temporal closeness and overlap of 
contextual cues between the encoding of Set 2 and the immediate recall, which increase the relative familiarity and distinctiveness of Set 2 objects (for a similar argument with regard to the time course of retroactive interference and false memory effects, see Howe, 1998).

In the group who recalled Set 2 instead of Set 1 in Session 3, very few intrusions from Set 1 into Set 2 were observed. This is especially interesting from a developmental perspective, because children 4 to 8 years of age show source discrimination difficulties in a variety of paradigms (e.g., Cycowicz et al., 2001; Drummey \& Newcombe, 2002). Therefore, the "unidirectional" intrusion effect found in Experiment 2 suggests that source discrimination problems are different from memory-updating processes (for further empirical testing and discussion of source discrimination problems in the reconsolidation paradigm, see Hupbach et al., 2009). Interestingly, Frost, Ingraham, and Wilson (2002) found similar asymmetrical source errors in a misinformation study. After a delay (but not when tested immediately), the majority of source attributions of misinformation details were incorrect (attributed to the event), whereas the majority of source attributions of event details were correct. While this and our findings cannot be readily accounted for within the source-monitoring framework, the memory reconsolidation account and TCM provide explanations of this unique error pattern.

\section{General discussion}

The primary purpose of the present set of studies was to further examine the role of spatial context in triggering episodic memory reconsolidation and updating. Our previous work had shown that spatial context was both necessary and sufficient, but these experiments were all carried out in contexts with which the participants had little prior experience. We speculated that this might not be the case when familiar spatial contexts were used, and tested this hypothesis in a series of studies with 5-year-old children. We chose this age because it was easy to manipulate context familiarity in this sample; we were able to use both a highly familiar context (the home) and a relatively unfamiliar context (a seldom-visited area at the children's day care center). Additionally, we were interested in seeing whether, and how much, memory updating occurs in children at this age.

Our results were quite clear: the critical role of spatial context in triggering memory updating that we observed when we used unfamiliar contexts (in adults) was not observed when we used familiar contexts in the 5-year-olds (Exp. 2A: context reminder). This failure did not occur because 5 -year-old children fail to show episodic memory updating. Indeed, 5-year-old children show clear updating effects (Exps. 1, 2A), and these effects are not due to general source memory problems (Exp. 2B: Set 2 recall). Furthermore, the effects emerge over time, consistent with the idea that reconsolidation requires the synthesis of new proteins (Exp. 2B: immediate recall). In this sense, the 5-year-olds behave just like adults when unfamiliar contexts are employed (Hupbach et al., 2007).

Why does familiarity have such an impact on the role played by context in memory reactivation and updating? At an intuitive level it seems obvious - an unfamiliar context can readily reactivate only the target memory under study, whereas a familiar context might reactivate any of many memories. In our situation, it is not surprising that a test context, never experienced by our participants, would reactivate the memory of the unique experience the participants had in that spatial context-learning a set of objects. Nor is it surprising that a familiar spatial context might fail to reactivate this target memory. This is especially true when other relevant features of the objectset learning procedure, namely the experimenter and the way in which the objects are presented, vary from Session 1 to Session 2. As we observed, when these other features are present prior to learning the second set, we again observe memory reactivation followed by updating. Thus, memory updating following reactivation is a relatively ubiquitous phenomenon, but reactivation is triggered by different cues depending on the test situation. While an unfamiliar spatial context appears to "overshadow" other cues, such as the experimenter and details of the procedure, this is not the case for familiar spatial contexts. We lack a clear understanding of the mechanism that underlies these overshadowing effects.

Comparable effects have been observed in other situations, including latent inhibition in rats, where Hall et al. (2009) have shown that familiar spatial contexts fail to initiate memory reactivation in taste aversion experiments. They concluded that preexposure to the context can retard conditioning to that context. However, in fear conditioning experiments, Fanselow has shown exactly the opposite effect: Exposure to the context is essential to observe contextual fear at all (e.g., Fanselow 1990; see Fanselow, 2007, for a review).

Whatever the basis of this shifting role of context, the ubiquitous nature of memory updating, and its presence in 5-year-olds as well as in adults, supports the emerging view that memories are malleable and subject to modification when conditions allow. Our results show that the trigger to such updating may vary from situation to situation, and it now becomes important to explore the range of triggering features and the conditions under which they are most effective. Memory reconsolidation as a principle might underlie the reported updating effects. However, the reconsolidation account is largely a neurobiological model based on animal data, and it does not describe the cognitive 
mechanisms involved in memory updating. The temporal context model provides such a mechanistic account and has been successfully applied to our previous adult data (Sederberg et al., 2011). However, this model does not address the role of spatial context in triggering our effects. The present study reveals two important facts about spatial context and memory updating that bear on this model: First, spatial context plays a primary role only when it is relatively novel. In familiar spatial contexts, such as one's home, other aspects of the situation, such as the experimenter and a reminder question, assume this primary role. Second, the predictions of the model with regard to output order, which seem fundamental to the model as presently configured, were only supported in the condition in which spatial context played no role in memory updating (the familiar-context case). When spatial context played a critical role (the unfamiliar-context case), output order did not conform to the model's predictions. Our results suggest that TCM, and related models that draw no distinction between different kinds of contextual cues, will need to reconsider this stance.

Author Note We thank Jennie Curcio, Auna Otts, Rabiah Muhammad, and Cong Zhang for help with data collection, and Kenneth Norman and two anonymous reviewers for their insightful comments. This research was funded by NSF Grant BCS-0743988 to L.N., A.H., and R.G., and NSF CAREER Award BCS-0238584 to R.G.

\section{References}

Anderson, J. R. (1974). Retrieval of propositional information from long-term memory. Cognitive Psychology, 6, 451-474.

Bjork, R. A., \& Whitten, W. B. (1974). Recency-sensitive retrieval processes in long-term free recall. Cognitive Psychology, 6, 173189. doi:10.1016/0010-0285(74)90009-7

Brainerd, C. J., Reyna, V. F., \& Ceci, S. J. (2008). Developmental reversals in false memory: A review of data and theory. Psychological Bulletin, 134, 343-382.

Bright-Paul, A., Jarrold, C., \& Wright, D. B. (2005). Age-appropriate cues facilitate source-monitoring and reduce suggestibility in 3 to 7-year-olds. Cognitive Development, 20, 1-18.

Bruck, M., \& Ceci, S. J. (1999). The suggestibility of children's memory. Annual Review of Psychology, 50, 419-439.

Cycowicz, Y. M., Friedman, D., Snodgrass, J. G., \& Duff, M. (2001). Recognition and source memory for pictures in children and adults. Neuropsychologia, 39, 255-267. doi:10.1016/S0028-3932 (00)00108-1

Drummey, A. B., \& Newcombe, N. S. (2002). Developmental changes in source memory. Developmental Science, 5, 502-513. doi:10.1111/1467-7687.00243

Dudai, Y. (2006). Reconsolidation: The advantage of being refocused. Current Opinion in Neurobiology, 16, 174-178.

Fanselow, M. S. (1990). Factors governing one-trial contextual conditioning. Animal Learning \& Behavior, 18, 264-270.

Fanselow, M. S. (2007). Context: What's so special about it? In H. L. Roediger III, Y. Dudai, \& S. M. Fitzpatrick (Eds.), Science of memory: Concepts (pp. 101-105). Oxford: Oxford University Press.
Frost, P. J., Ingraham, M., \& Wilson, B. (2002). Why misinformation is more likely to be recognized over time: A source monitoring account. Memory, 10, 179-185.

Hall, G., \& Symonds, M. (2006). Overshadowing and latent inhibition of context aversion conditioning in the rat. Autonomic Neuroscience, $129,42-49$.

Hall, G., Symonds, M., \& Rodriguez, M. (2009). Enhanced latent inhibition in context aversion conditioning. Learning and Motivation, 40, 62-73. doi:10.1016/j.lmot.2008.05.001

Hardt, O., Einarsson, E. Ö., \& Nader, K. (2010). A bridge over troubled water: Reconsolidation as a link between cognitive and neuroscienctific memory research traditions. Annual Review of Psychology, 61, 141-167. doi:10.1146/annurev.psych.093008. 100455

Howard, M. W., \& Kahana, M. J. (1999). Contextual variability and serial position effects in free recall. Journal of Experimental Psychology: Learning, Memory, and Cognition, 25, 923-941. doi: $10.1037 / 0278-7393.25 .4 .923$

Howard, M. W., \& Kahana, M. J. (2002). A distributed representation of temporal context. Journal of Mathematical Psychology, 46, 269-299. doi:10.1006/jmps.2001.1388

Howe, M. L. (1995). Interference effects in young children's long-term retention. Developmental Psychology, 31, 579-596.

Howe, M. L. (1998). When distinctiveness fails, false memories prevail. Journal of Experimental Child Psychology, 71, 170 177.

Howe, M. L., \& Brainerd, C. J. (1989). Development of children's long-term retention. Developmental Review, 9, 301-340. doi:10.1016/0273-2297(89)90032-4

Hupbach, A., Gomez, R., Hardt, O., \& Nadel, L. (2007). Reconsolidation of episodic memories: A subtle reminder triggers integration of new information. Learning \& Memory, 14, 4753. doi: $10.1101 / \mathrm{lm} .365707$

Hupbach, A., Gomez, R., \& Nadel, L. (2009). Episodic memory reconsolidation: Updating or source confusion? Memory, 17, 502-510. doi:10.1080/09658210902882399

Hupbach, A., Hardt, O., Gomez, R., \& Nadel, L. (2008). The dynamics of memory: Context-dependent updating. Learning \& Memory, 15, 574-579. doi:10.1101/1m.1022308

Hupbach, A., Weiss, M., Gomez, R., \& Nadel, L. (2011). Memory updating is modulated by memory strength. Manuscript in preparation.

Johnson, M. K., Hashtroudi, S., \& Lindsay, D. S. (1993). Source monitoring. Psychological Bulletin, 114, 272-277.

Koppenaal, R. J., Krull, A., \& Katz, H. (1964). Age, interference, and forgetting. Journal of Experimental Child Psychology, 1, 360375.

Lee, K., \& Bussey, K. (2001). Children's susceptibility to retroactive interference: The effects of age and degree of learning. Journal of Experimental Child Psychology, 80, 372-391. doi:10.1006/ jecp. 2001.2638

Lever, C., Burton, S., Jeewajee, A., Wills, T. J., Cacucci, F., Burgess, N., et al. (2010). Environmental novelty elicits a later theta phase of firing in CA1 but not subiculum. Hippocampus, 20, 229-234.

Loftus, E. F. (2005). Planting misinformation in the human mind: A 30 -year investigation of the malleability of memory. Learning \& Memory, 12, 361-366. doi:10.1101/lm.94705

Nader, K. (2003). Memory traces unbound. Trends in Neurosciences, 26, 65-72.

Postman, L., \& Underwood, B. J. (1973). Critical issues in interference theory. Memory \& Cognition, 1, 19-40.

Roberts, K. P. (2002). Children's ability to distinguish between memories from multiple sources: Implications for the quality and accuracy of eyewitness statements. Developmental Review, $22,403-435$. 
Sederberg, P. B., Gershman, S. M., Polyn, S. M., \& Norman, K. A. (2011). Human memory reconsolidation can be explained using the temporal context model. Psychonomic Bulletin \& Review, 18, 455-468

Sederberg, P. B., Howard, M. W., \& Kahana, M. J. (2008). A contextbased theory of recency and contiguity in free recall. Psychological Review, 115, 893-912. doi:10.1037/a0013396

Smith, S. M., \& Vela, E. (2001). Environmental context-dependent memory: A review and meta-analysis. Psychonomic Bulletin \& Review, 8, 203-220.
Tronson, N. C., \& Taylor, J. R. (2007). Molecular mechanisms of memory reconsolidation. Nature Reviews Neuroscience, 8, 262275. doi: $10.1038 / \mathrm{nrn} 2090$

Wang, S. H., de Oliveira Alvares, L., \& Nader, K. (2009). Cellular and systems mechanisms of memory strength as a constraint on auditory fear reconsolidation. Nature Neuroscience, 12, 905-912.

Watkins, O. C., \& Watkins, M. J. (1975). Buildup of proactive inhibition as a cue-overload effect. Journal of Experimental Psychology: Human Learning and Memory, 1, 442-452. doi:10.1037/0278-7393.1.4.442 